\title{
Video-microscopic imaging of cell spatio-temporal dispersion and migration
}

Christine Terryn, Arnaud Bonnomet, Jérôme Cutrona, Christelle Coraux, Jean-Marie Tournier, Béatrice Raby-Nawrocki, Myriam Polette, Philippe Birembaut and JeanMarie Zahm

INSERM, U903, Reims, F-51092, France ; IFR53, Reims, F-51095, France ; Univ Reims Champagne Ardenne, Reims, F-51095, France ; CHU Reims, Hôpital Maison Blanche, Reims, F-51092, France.

\section{Table of content}

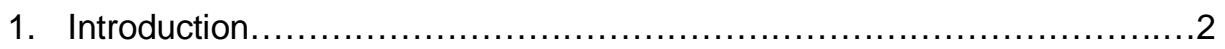

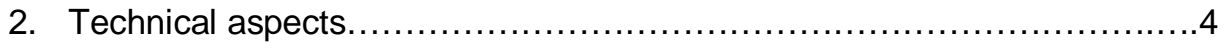

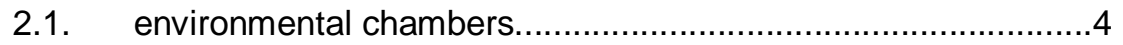

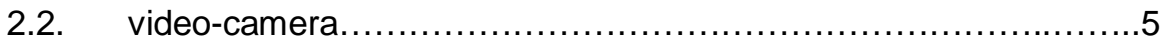

2.3. microscope automatisation......................................... 6

3. Two and three-dimensional analysis of cell behavior........................ 8

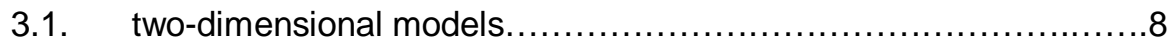

3.1.1 sparse cultures...............................................

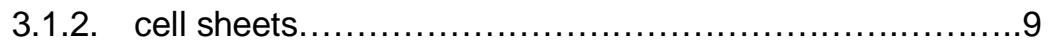

3.2. three-dimensional models......................................... 10

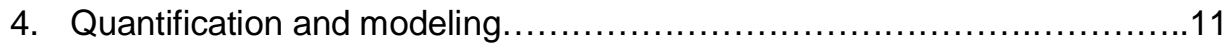

4.1. cell migration analysis............................................... 11

4.2. cell dispersion analysis........................................... 12

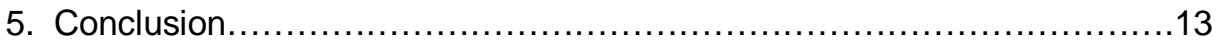



\section{Abstract}

Live-cell imaging has become a powerful analytical tool in most cell biology laboratories. The scope of this paper is to give an overview of the environmental considerations for maintaining living cells on the microscope stage and the technical advances permitting multi-parameter imaging. The paper will then focus on two-dimensional and three-dimensional analysis of cell dispersion and migration and finally give a brief insight on computational modeling of the cell behavior.

\section{Keywords}

Video-microscopy, live-cell imaging, cell migration, cancer, wound repair.

\section{Biography of the corresponding author}

Jean-Marie Zahm took his PhD in 1989 and his Research Habilitation in 1994. He is a INSERM research engineer. His scientific expertise concerns the functionality of the airway epithelium and dynamic cellular imaging.

\section{Corresponding author}

Jean-Marie Zahm

INSERM UMRS 903, 45 rue Cognacq-Jay, 51092 Reims cedex, France

Email:jm.zahm@univ-reims.fr 


\section{Introduction}

The development of cellular biology has been for a long time dependent on the witness to describe cellular function through static images of cells, without taking in consideration the time-dependent fluctuations. To date, an increasing number of investigations are using live-cell imaging to provide insight into the nature of cellular and tissue functions, especially due to the rapid advances that occur in microscopy technology. Being able to observe processes as they happen within the cell by light microscopy adds a vital extra dimension to our knowledge of cell function. Because of these advances, live-cell imaging has become a powerful analytical tool in most cell biology laboratories. With the advanced digital camera systems available to date, the increasingly popular technique of live-cell imaging is becoming broadly applied to record living cells over periods ranging from milliseconds to several days or weeks. First, the digital camera is a patient and methodical observer that watches over a microscopic field with the wanted details (magnification and resolution) and the determined watchfulness (time-lapse between 2 successive images). Live-cell imaging requires the control of space (amount of information). The recording system must discriminate through the microscope most of the details at the cellular level. In video-microscopy, the image is typically projected by the optical system onto the surface of an electric image sensor such as a CCD (charge coupled device) camera. To optimize the information content of the resulting image, the resolution of the detector must closely match that of the microscope. Live-cell imaging also requires the control of time. In cellular biology, most of the cell modifications avoid our 
perception: we do not have time to collect the necessary information, we lose visual memory of a previous state localized too far in the time scale. The memory of the events occurring in cells is now constituted by the computer to which is connected the digital camera. Repeated imaging of a cell culture at defined time points provides information on dynamic processes that often occur with a wide distribution of time scales.

Imaging living cells with transmitted light is often used to provide information on cell shape, position and motility. During morphogenesis, failure of cells to migrate, or migration of cells to inappropriate locations, can result in life threatening consequences. In the adult, cell migration is central to homeostatic processes such as the effective immune response and the repair of injured tissues. Furthermore, it contributes to pathologies, including vascular disease, chronic inflammatory diseases, tumor formation and metastasis. Only a small proportion of highly specialized cells are able to actively and autonomously move within the organism: stem cells, leukocytes, fibroblasts, sperm cells and tumor cells. Multi-cell migration often occurs in biology [1]. Coordinated cellular movements allow tissue formation during embryogenesis and the repair of tissue after injury. Metastatic cells can migrate as a group [2]. The biologist must therefore develop in vitro models mimicking the in vivo behavior of cells and the physicists must improve microscope systems and software. These contributions have led to unprecedented access to sophisticated imaging technology.

We will successively give an overview of the environmental considerations for maintaining cell viability and functions on the microscope stage and the technical advances permitting multi-parameter imaging. We will then focus on two-dimensional 
and three-dimensional analysis of cell motility and finally give a brief insight on computational modeling of cell to cell interaction and cell motility.

\section{Technical aspects}

Live-cell microscopy represents a compromise between achieving the best possible image quality and preserving the cell viability. Dedicated sophisticated microscopes should be used to perform this type of microscopy. Microscope manufacturers have invested in the development of live-cell microscopy and currently, there are, at least 4 available patterns: Eclipse TE 2000-PFS by Nikon (Nikon Instrument INC, USA), Axiovert 200 by Zeiss (Carl Zeiss SAS, Germany) AF 6000 by Leica (Leica Microsystems, Germany) and Cell M by Olympus (Olympus, Germany).

\subsection{Environmental chambers}

The first condition for performing videomicroscopy is to maintain live-cells on the microscope stage. Indeed, cells culture must be maintained in conditions that promote growth and normal function to avoid potential artifacts in the interpretation of experimental results. Videomicroscopes are equipped with environmental chambers where physical parameters like temperature, humidity or gas mixture are controlled. Generally, local specimen temperature is maintained at $37^{\circ} \mathrm{C}$ and the humidity degree of the atmosphere is around $97 \%$. To keep cells medium $\mathrm{pH}$ around 7 , the atmosphere around the specimen contains $5 \%$ of carbon dioxide.

Two types of environmental chamber can be coupled with the videomicroscope: open chambers or closed chambers. Open chambers (PeCon GmbH, Germany) allow the use of classical Petri dishes or multi-well dishes and permit a free access to the atmosphere, easy microinjection, addition of drugs, culture medium change or 
other manipulations of the cells. The closed chamber (Bioptechs, Pennsylvania) shows a better isolation from the external environment but the access to the cells is more difficult. This system allows addition of fresh medium and drugs during the experiment without interrupting the imaging sequence. Likewise, both live-cell chambers can present excellent optical properties and are coupled with a metal heating plate under thermistor control. Indeed, cellular functions are extremely sensitive to temperature variations. A small change in temperature can imply profound effects on cell physiology. Concerning the microscope, temperature changes can produce unwanted movement in the optical path that can induce focus or alignment shifts. The heating plate is adapted to different sort of specimen mountings from Petri dish to multi-well plates.

\subsection{Video-camera}

The second condition for live-cell imaging is to achieve the best possible image quality. Indeed, the user must determine the most important parameter to optimize the acquisition between illumination duration and optimal signal recording. To acquire the images, the video-microscopes are usually equipped with a CDD (Coupled Charge Device) camera. This type of detector exhibits a dark current associated with each pixel but alternative devices, including cooled devices to very low temperature, lead to the improvement of camera sensitivity. However, an additional noise known as "read noise" due to each pixel read and conversion of analog signal to digital signal, can lead to low quality images mainly when low illumination time are required. Fortunately, modern CCD camera can be configured to deliver a significant increase in sensitivity by using a process known as "binning". During normal readout, the pixels in a CCD are measured by transferring a row of horizontal pixels into the read register, which is then sequentially analyzed. In a binned image, the pixels are 
combined by adjacent blocks (such as a $2 \times 2$ or $4 \times 4$ block) and the total value of the block is assigned to an image pixel. Thus, if a $2 \times 2$ binning is used, the recorded signal is four-fold increased and the signal-to-noise ratio is two-fold improved. However, the binning procedure induces a loss in resolution (two-fold loss in the case of $2 \times 2$ binning).

No single camera will meet all requirements in digital microscopy, so the investigator must compromise. Choosing the most appropriate detector for an experiment depends on the light level to be detected and the resolution goal of the project. For transmitted light microscopy, a simple CCD detector would suffice. For fluorescence microscopy, the investigator has the choice between a cooled CCD camera or an intensified CCD (ICCD). The ICCD is characterized by high speed and sensitivity, but by limited dynamic range and resolution. The cooled CCD has high quantum efficiency, large dynamic range and excellent spatial resolution. In order to overcome the shortcomings of these conventional CCD cameras, the manufacturers have introduced an innovative method for amplifying weak signals. Electron multiplying devices (EMCCD) achieve the sensitivity of ICCD without compromising the quantum efficiency and resolution characteristics of a conventional CCD. In the end, the user must define an optimal choice to improve the image quality without dramatic loss of resolution.

\subsection{Microscope automatisation}

The videomicroscope can be totally automated and becomes a powerful instrument to investigate living cells, mainly to process time-lapse experiments. Indeed, image acquisition can be performed in a time range from a few milliseconds to 24 or 48 hours. All the components such as shutters on the light path and the motorized stage of the microscope can be driven by a host computer through 
commercially available image acquisition software. When using multi-well plates, several positions can be programmed using a "position list" menu. Therefore the experimenter can follow, during the same acquisition sequence, the cell behavior in different conditions. For example, some drugs can be added in different concentrations in each well, that allows to follow cell migration in each condition during the same image acquisition. Another experiment consists in following the cell migration when different types of drugs are added or to study the cell behavior at the same time for different cell lines (for example normal or cancerous cell lines).

Moreover, the software pilots the electromechanical shutter on the transmission or epi-fluorescence light paths. Then, during the time-lapse experiment, the live cells are only illuminated during the necessary exposition time to take photography and not for all the time of the time-lapse experiment. In these conditions, the illumination does not cause some temperature rise or photodamage and therefore avoids perturbation in the functionality of living cells.

Another parameter is important to consider if the investigator wants to extend imaging from two-dimensional studies to three-dimensional studies. This parameter concerns the possibility to change the focus, allowing to record images from multiple focal planes. The software must give the possibility to define a different value of Zfocus at each well of the culture plate. Then, the acquisition can be programmed between a lower limit position and an upper limit position with a given step between each Z-level.

The videomicroscope allows also the acquisition of images in fluorescence microscopy mode. A wide variety of bandpass filters and dichromatic mirror combinations, designed to image a single fluorophore or to enable simultaneous 
imaging of specimens labeled with two or more fluorescent probes, are available from the microscope manufacturers. The acquisition software must drive a device for rapidly switching between different wavelengths of light through the use of multiple filter sets, monochromators or acousto-optic tunable filters. Recently, a new illumination system using LEDs (Light Emitting Diode) (Colibri, Carl Zeiss SAS, Germany) has been developed instead of HBO lamp fluorescence source. Each individual LED delivers only a precisely defined range of the spectrum, with a precise adjustment of intensity for optimum protection of samples and switching times in the microsecond range. Other illumination systems like MT 20 by Olympus (Olympus, Germany) or EL 6000 by Leica (Leica Microsystems, Germany) are also dedicated to fast switch of fluorescence wavelengths. These improvements in imaging microscopy devices lead to the development of study of fast cellular phenomenon and may improve the image quality without inducing dramatic specimen photo bleaching.

Video microscope devices represent a powerful tool to study living cells but also generate a high amount of data. This is particularly the case in threedimensional experiments, for example when a z-stack of 200 planes, during $24 \mathrm{~h}$, including 1 image per hour over 12 wells, generates more than 20 Go (Giga Octets) of data. Storage data problem should not be neglected and a complete data storage solution must be associated to the videomicroscope.

\section{Two and three-dimensional analysis of cell motility}

Depending on physiological or pathological conditions, the cells are induced to modify their behavior for movement and interactions with neighbouring cells. To investigate cell behavior, particularly cell migration, researchers have developed a 
plethora of assays, each with advantages and disadvantages. Thus, before beginning the investigations, the goals of the experiments should be carefully considered and the appropriate assay therefore chosen.

\subsection{Two-dimensional models}

Most studies devoted to the analysis of cell migration in vitro utilize models in which the cells are plated on a glass or plastic surface pre-coated or not with extracellular matrix proteins like collagen type I, fibronectin [3], collagen type IV [4] or laminin [5]. These two-dimensional assays are easy to handle and provide valuable insight into the cell behavior in response to pharmacological modulators. Two relevant behaviors of cells can be analyzed in two-dimensional models: the cell motility in sparse cultures or in cell sheets.

\subsubsection{Sparse cultures}

To characterize the motility of individual cells and the cell to cell interactions, timelapse video microscopy of low density cultures can be conducted. As previously described, this model of cell dispersion [6;7] allows the analysis and the quantification of the spatial individual or collective behavior of cells. In addition to the measurement of individual cell motility, this original technique is also able to study concomitantly the cohesion level of cells and their individual ability to disperse. Typical images obtained with 2 different bronchial cell lines (16HBE, a non-invasive cell line and BZR, an invasive cell line) are shown in figure 1. After 2 hours of culture, no difference in the spatial distribution of the cell lines is observed: the cells are randomly distributed within the observation fields (Fig. 1a,b). Afterwards, the non-invasive cell line rapidly aggregates to form clusters of different sizes (Fig. 1c), whereas the invasive cell line remains randomly distributed throughout the time of culture (Fig. 1d). Interestingly, 
the spatial group behavior of cell lines, i.e., their cohesion/dispersion ability reflects their invasive properties [7], whereas the measurement of their migration speed might not allow to differentiate these cell lines [8]. These assays on sparse cultures are probably the most widespread and have contributed to the fundamental understanding of cell migration processes at the molecular level [9]. The molecular factors involved in these processes are numerous and closely interacting. Cell migration involves protrusion of the plasma membrane (lamellipodium extension) at the leading edge of the cell, which implies cytoskeleton reorganization such as actin [10] or vimentin, the major intermediate filament protein in mesenchymal cells [11]. Cell movement also implies the formation of new sites of adhesion to the extracellular matrix at the front of the cells but also the release of adhesion sites at the back of the cells. This necessarily implies an interaction with extracellular matrix proteins (ECM) with the help of adhesion molecules such as integrins [12] and metalloproteinases (MMPs) with a regulation between MMP and their inhibitors [13]. Furthermore, production of ECM by cells during the migration process requires signalling pathway through specific receptors (for growth factors) on the airway cell surface. The analysis of movement of single cells by imaging allows the understanding of these complexe processes and to correlate movement and shape changes of cells with the spatio-temporal dynamics of the cytoskeletal elements and the extra and intracellular pathways involved in cell behaviour.

cell sheets

Multi-cell migration as cell sheet is of great importance in tissue formation during embryogenesis, repair of tissue after injury or tumoral invasion [1]. Particularly relevant as a model of sheet migration by epithelia, the classic wound assay is useful 
for studying cell migration in vitro. In this assay, cells are grown on a two-dimensional surface until they reached confluence and a group of cells is then removed by mechanically scraping [14] or chemically desquamating [15] the cell layer. The monolayer responds with cell spreading and migration into the denuded area until the wound is closed (figure 2). Another way to obtain multi-cell migration consists in using explants outgrowth cultures. Explant outgrowths of animal or human epithelium dissected from various tissues are seeded on an extracellular matrix in culture medium [16]. After a few days, epithelial cells migrate in the outgrowth around the explants. An alternative migration-induction procedure, referred to as the ring system (figure 3), consists in plating the cells inside a small glass ring (6 to $10 \mathrm{~mm}$ in diameter) placed in the middle of a culture plate. At cell confluency, the ring is removed to allow cell migration from the confluent area initially delimited by the ring [17]. These assays are classic and commonly used methods for studying cell migration, particularly during wound repair, and the biology underlying it, i.e. the contribution of various cytokines, growth factors and extracellular matrix (ECM) molecules that may modulate the provisional matrix over which the cells migrate and that trigger behavioral changes in the cells during migration. Advances in cell biology have identified a variety of molecules, specifically growth factors and their receptors, which are critically involved in directing cell functions during soft tissue organogenesis. Interestingly, expression and functional relevance of most of these growth factor-receptor-systems have also been demonstrated in the repair response of various tissues [18]. 


\subsection{Three-dimensional models}

An increasing amount of experimental data shows that growing cells within threedimensional matrices reduces the gap between cell cultures and real tissues. Threedimensional cell cultures mimic in vitro cell-cell and cell-extracellular matrix interactions. The potential of three-dimensional cell culture was demonstrated by the finding that although nonmalignant and malignant epithelial cells were phenotypically similar under two-dimensional culture conditions, they behaved very differently when grown as three-dimensional cultures [19;20]. By employing time-lapse imaging of cells in collagen, Friedl et al [21] showed that invasive cancer cells easily change migration strategy. Three-dimensional cultures are currently applied to a broad range of investigations, including tumor biology or epithelial morphogenesis. The eligible application of three-dimensional assays is the analysis of the cell migration process. Cell are generally embedded within a three-dimensional matrix commonly made of collagen type-I [22]. In addition, this matrix can be placed on the membrane of the inner chamber of a double compartment culture chamber, thus allowing the addition of chemo-attractants to the basal part of the chamber [20]. To follow the migratory behavior of the cells in a three-dimensional environment, the method of choice is time-lapse videomicroscopy under bright field illumination. From these threedimensional assays, the individual migration paths of cells can be analyzed and allow the calculation of parameters such as speed of migration, persistence and directionality of migration (figure 4) $[9 ; 20 ; 23-26]$. The use of three-dimensional models of cell migration provides new insights, but because of the very thin and generally elongated morphology of cells in three-dimension, many cellular structures can appear less obvious than those observed in flattened cells on two-dimensional substrates. There is therefore a need for better optical resolution in time-lapse 
imaging. Single plane illumination microscopy (SPIM) is a new imaging method allowing high spatial resolution with minimal photodamage and will allow the study of subcellular processes in large living specimens [27].

Molecules involved in cancer cell migration could be potential targets for antimetastatic therapy. The use of three-dimensional models has revealed new aspects of cancer cell migration and focused our attention on specialized cell protusions involved in chemotaxis and extracellular matrix invasion and will lead to the development of drugs that target cell motility [28].

\section{Quantification and modeling}

The quantification of the motile behavior of cells relies not only on the choice of appropriate in vitro experimental models, but also on the analysis of the huge amount of data obtained through time-lapse videomicroscopy. It is also of interest to manage these data by relevant mathematical models.

\section{1. cell migration analysis}

Depending on the biological model, cell migration can be analyzed by different methods. The motility of cells in sparse cultures is generally measured by means of custom cell-tracking algorithms to acquire trajectories of several tens of crawling cells $[1 ; 29]$. However automatic tracking procedures are not reliably able to cope with dense populations of cells located very close to each other, especially when nondirected migration is involved. Thus the investigators often rely on interactive tracking of cells rather than on automatic tracking [29]. This is a very tedious procedure. Simplest procedures are used when analyzing cell migration from global population 
analysis such as in the wound healing models. From the time-lapse images, the wound margin can be drawn manually or by iterative image segmentation to determine the evolution with time of the wounded area [30]. Methods allowing the quantification of the cell migratory behavior of a whole cell population have been described. Ronot et al [31] proposed an approach based on an optical flow analysis that consists in calculating the velocity field over a cell monolayer, thus providing a quantification of both temporal variation of cell velocity and spatiotemporal reorganization of the cell population within the monolayer, without previous cell labeling. More recently, we used image cross-correlation microscopy for characterizing the migration properties of cell populations [32]. Computer-assisted cell tracking has also been introduced for the reconstruction of individual cell trajectories in 3D assays [20;33;34].

\section{2. cell distribution analysis}

Understanding the relationship between cell cohesion and cell invasion could help in elucidating mechanisms contributing to the acquisition by cells of an invasive phenotype. We have developed a model that allowed the analysis and quantification of the spatial collective behavior of different epithelial cell lines [7]. This model, based on the use of graphical geometrical methods, presents the advantage of allowing the dynamic study of the spatiotemporal behavior of living cells. Interestingly, these graphical methods can yield parameters characterizing the cohesion level of cells and their individual ability to disperse and to be invasive (figure 5). One should conclude from these data that the dynamic clustering formation of the non invasive cells could be based on random movement and molecular adhesion properties at the cellular level. This later hypothesis is emphasized by the results reported by 
Nawrocki-Raby et al [7], demonstrating that the modulation of E-cadherin expression induced modifications of the cell spatial distribution. Taken together, all these data suggest that the spatial group behavior of cells lines is rather dependent on the capacity of cells to adhere each other and to remain strongly adherent, than to be governed by a directed motion of the cells.

\section{Conclusion}

During the last decade substantial improvement in video-microscopic techniques occurred, providing a real-time view of dynamic processes at the cellular level. The automation of spatio-temporal imaging and the investigation of multiple samples in a single experiment highly increase the amount of data obtained in a research department and is key to the understanding of biological phenomenons. The development of fluorescent biosensor and the use of more sophisticated in vitro models such as 3D culture systems would also greatly improve this understanding. 


\section{References}

1. Bindschadler M, McGrath JL. Sheet migration by wounded monolayers as an emergent property of single-cell dynamics. J. Cell Sci. 2007: 120: 876-84.

2. Hegerfeldt $Y$, Tusch $M$, Brocker EB, Friedl $P$. Collective cell movement in primary melanoma explants: plasticity of cell-cell interaction, beta1-integrin function, and migration strategies. Cancer Res. 2002: 62: 2125-30.

3. Newgreen DF, Gibbins IL, Sauter J, Wallenfels B, Wutz R. Ultrastructural and tissue-culture studies on the role of fibronectin, collagen and glycosaminoglycans in the migration of neural crest cells in the fowl embryo. Cell Tissue Res. 1982: 221: 521-49.

4. Masiero L, Lapidos KA, Ambudkar I, Kohn EC. Regulation of the RhoA pathway in human endothelial cell spreading on type IV collagen: role of calcium influx. J. Cell Sci. 1999: 112 ( Pt 19) : 3205-13.

5. Donaldson DJ, Mahan JT. Epidermal cell migration on laminin-coated substrates. Comparison with other extracellular matrix and non-matrix proteins. Cell Tissue Res. 1984: 235: 221-4.

6. Abercrombie M, Heaysman JE. Observations on the social behaviour of cells in tissue culture. II. Monolayering of fibroblasts. Exp. Cell Res. 1954: 6: 293306.

7. Nawrocki RB, Polette M, Gilles C et al. Quantitative cell dispersion analysis: new test to measure tumor cell aggressiveness. Int. J. Cancer 2001: 93: 64452.

8. Matos M, Raby BN, Zahm JM et al. Cell migration and proliferation are not discriminatory factors in the in vitro sociologic behavior of bronchial epithelial cell lines. Cell Motil. Cytoskeleton 2002: 53: 53-65.

9. Entschladen F, Drell TL, Lang K et al. Analysis methods of human cell migration. Exp. Cell Res. 2005: 307: 418-26.

10. Carlier MF, Pantaloni D. Control of actin assembly dynamics in cell motility. $J$. Biol. Chem. 2007: 282: 23005-9.

11. Ivaska J, Pallari HM, Nevo J, Eriksson JE. Novel functions of vimentin in cell adhesion, migration, and signaling. Exp. Cell Res. 2007: 313: 2050-62. 
12. Chan KT, Cortesio CL, Huttenlocher A. Integrins in cell migration. Methods Enzymol. 2007: 426: 47-67.

13. Polette M, Nawrocki-Raby B, Gilles C, Clavel C, Birembaut P. Tumour invasion and matrix metalloproteinases. Crit Rev. Oncol. Hematol. 2004: 49: 179-86.

14. Yarrow JC, Totsukawa G, Charras GT, Mitchison TJ. Screening for cell migration inhibitors via automated microscopy reveals a Rho-kinase inhibitor. Chem. Biol. 2005: 12: 385-95.

15. Zahm JM, Kaplan $\mathrm{H}$, Herard AL et al. Cell migration and proliferation during the in vitro wound repair of the respiratory epithelium. Cell Motil. Cytoskeleton 1997: 37: 33-43.

16. Chevillard M, Hinnrasky J, Zahm JM, Plotkowski MC, Puchelle E. Proliferation, differentiation and ciliary beating of human respiratory ciliated cells in primary culture. Cell Tissue Res. 1991: 264: 49-55.

17. Gilles $C$, Polette M, Zahm JM et al. Vimentin contributes to human mammary epithelial cell migration. J. Cell Sci. 1999: 112 ( Pt 24) : 4615-25.

18. Werner S, Grose R. Regulation of wound healing by growth factors and cytokines. Physiol Rev. 2003: 83: 835-70.

19. Petersen OW, Ronnov-Jessen L, Howlett AR, Bissell MJ. Interaction with basement membrane serves to rapidly distinguish growth and differentiation pattern of normal and malignant human breast epithelial cells. Proc. Natl. Acad. Sci. U. S. A 1992: 89: 9064-8.

20. Hazgui S, Bonnet $\mathrm{N}$, Cutrona J et al. 3D culture model and computer-assisted videomicroscopy to analyze migratory behavior of noninvasive and invasive bronchial epithelial cells. Am. J. Physiol Cell Physio/ 2005: 289: C1547-C1552.

21. Friedl $P$, Wolf $K$. Tumour-cell invasion and migration: diversity and escape mechanisms. Nat. Rev. Cancer 2003: 3: 362-74.

22. Hooper S, Marshall JF, Sahai E. Tumor cell migration in three dimensions. Methods Enzymol. 2006: 406: 625-43.

23. Friedl $P$, Brocker EB. Reconstructing leukocyte migration in $3 D$ extracellular matrix by time-lapse videomicroscopy and computer-assisted tracking. Methods Mol. Biol. 2004: 239: 77-90. 
24. Amler RW, Barone S Jr, Belger A et al. Hershey Medical Center Technical Workshop Report: optimizing the design and interpretation of epidemiologic studies for assessing neurodevelopmental effects from in utero chemical exposure. Neurotoxicology 2006: 27: 861-74.

25. Horobin AJ, Shakesheff KM, Pritchard DI. Promotion of human dermal fibroblast migration, matrix remodelling and modification of fibroblast morphology within a novel 3D model by Lucilia sericata larval secretions. J. Invest Dermatol. 2006: 126: 1410-8.

26. Sun $\mathrm{S}$, Wise J, Cho M. Human fibroblast migration in three-dimensional collagen gel in response to noninvasive electrical stimulus. I. Characterization of induced three-dimensional cell movement. Tissue Eng 2004: 10: 1548-57.

27. Verveer PJ, Swoger J, Pampaloni F et al. High-resolution three-dimensional imaging of large specimens with light sheet-based microscopy. Nat. Methods 2007: 4: 311-3.

28. Yamaguchi H, Wyckoff $\mathrm{J}$, Condeelis J. Cell migration in tumors. Curr. Opin. Cell Biol. 2005: 17: 559-64.

29. Matos M, Raby BN, Zahm JM et al. Cell migration and proliferation are not discriminatory factors in the in vitro sociologic behavior of bronchial epithelial cell lines. Cell Motil. Cytoskeleton 2002: 53: 53-65.

30. Rosello C, Ballet P, Planus E, Tracqui P. Model driven quantification of individual and collective cell migration. Acta Biotheor. 2004: 52: 343-63.

31. Ronot X, Doisy A, Tracqui P. Quantitative study of dynamic behavior of cell monolayers during in vitro wound healing by optical flow analysis. Cytometry 2000: 41: 19-30.

32. Bonnet N, Delavoie F, Zahm JM. Characterizing the spatio-temporal behavior of cell populations through image auto- and cross-correlation microscopy. Biotechniques 2007: 43: 107-15.

33. Friedl P, Brocker EB. The biology of cell locomotion within three-dimensional extracellular matrix. Cell Mol. Life Sci. 2000: 57: 41-64.

34. Niggemann B, Drell TL, Joseph J et al. Tumor cell locomotion: differential dynamics of spontaneous and induced migration in a 3D collagen matrix. Exp. Cell Res. 2004: 298: 178-87. 


\section{Figure captions}

Figure 1: spatio-temporal repartition of an non-invasive (16HBE: a,c) and invasive (BZR: b, d) cell line two hours after seeding $(a, b)$ and after $24 \mathrm{~h}$ of culture (c,d). Two hours after seeding, both cell lines are randomly distributed within the observation field, but after $24 \mathrm{~h}$ of culture, the non-invasive cell line aggregates to form clusters, whereas the invasive cell line remains randomly distributed throughout the time of culture.

Figure 2: In vitro model of wound repair. Airway epithelial cells dissociated from human nasal polyps are cultured up to confluency. The culture medium is then removed and a $2 \mu \mathrm{l}$ drop of $\mathrm{NaOH} 1 \mathrm{~N}$ is deposited for 2 sec on the center of the culture to produce cellular desquamation; the cellular debris are then rapidly rinsed with fresh culture medium (a).The cell monolayer responds with cell spreading and migration (b) into the denuded area until the wound is closed (c). Images were recorded at $2 \mathrm{hr}$ intervals.

Figure 3: in vitro model of cell migration. Airway epithelial cells in primary culture are seeded inside a small glass ring placed in the middle of a culture plate and cultured up to confluency (a). The glass ring is then removed to allow cell migration (b). Cells migrate from the confluent area initially delimited by the ring (c). A typical migratory cell phenotype is observed at the periphery of the culture (d). 
Figure 4: Image of a non-invasive cell line (16HBE: a) and an invasive cell line (BZR: b) cultured within a three-dimensional collagen gel. Images were recorded every hour for $24 \mathrm{hr}$ at 100 different focus planes ( $3 \mu \mathrm{m}$ between each plane) and the cell trajectories were computed from these images. Panel $c$ and $d$ show the threedimensional representation of the trajectories of the non-invasive cell line (c) and the invasive cell line (d). Each colour on panel $c$ and d corresponds to the trajectory of different cells.

Figure 5: illustration of the spatial repartitions observed for a non-invasive (16HBE: a) and an invasive (BZR: b) cell line. From the images in figure 1, graphical methods (Voronoï's diagram) are computed from the different spatial cell positions. Quantitative descriptors calculated from these graphical representations allow differentiate the spatial repartition of cells according to their invasive capacity. 